\title{
Sepiapterin Enhances Tumor Radio- and Chemosensitivities by Promoting Vascular Normalization
}

\author{
Christopher S. Rabender, Ninu Bruno, Asim Alam, Gobalakrishnan Sundaresan, Jamal Zweit, \\ and Ross B. Mikkelsen
}

Department of Radiation Oncology (C.S.R., N.B., A.A., R.B.M.) and Center for Molecular Imaging (G.S., J.Z.), Virginia

Commonwealth University, Richmond, Virginia

Received October 19, 2017; accepted February 26, 2018

\begin{abstract}
Previously, we demonstrated that nitric oxide (NO) synthase (NOS) is uncoupled in a wide range of solid tumors and that restoring NOS coupling with the tetrahydrobiopterin precursor sepiapterin (SP) inhibits tumor progression. Endothelial dysfunction characterizes the poorly functional vasculature of solid tumors, and since NO is critical for regulation of endothelial function we asked whether SP, by recoupling NOS, improves tumor vasculature structure and function-enhancing chemotherapeutic delivery and response to radiotherapy. MMTV-neu mice with spontaneous breast tumors were treated with SP by oral gavage and evaluated by multispectral optoacoustic tomographic analysis of tumor $\mathrm{HbO}_{2}$ and by tissue staining for markers of hypoxia, blood perfusion, and markers of endothelial and smooth muscle proteins. Recoupling tumor NOS activity
\end{abstract}

results in vascular normalization observed as reduced tumor hypoxia, improved tumor percentage of $\mathrm{HbO}_{2}$ and perfusion, as well as increased pericyte coverage of tumor blood vessels. The normalized vasculature and improved tumor oxygenation led to a greater than 2 -fold increase in radiationinduced apoptosis compared with radiation or SP alone. High-performance liquid chromatography analysis of tumor doxorubicin levels showed a greater than $50 \%$ increase in doxorubicin uptake and a synergistic effect on tumor cell apoptosis. This study highlights for the first time the importance of NOS uncoupling and endothelial dysfunction in the development of tumor vasculature and presents a new approach for improving the tumoricidal efficacies of chemotherapy and radiotherapy.

\section{Introduction}

The abnormal structure and function of tumor vasculature is well documented (Carmeliet and Jain, 2011). An imbalance in proangiogenic and antiangiogenic signaling stimulating the overproduction of angiogenic signals and aberrant endothelial cells leads to uneven, heterogeneous blood flow, and leaky, hemorrhagic blood vessels lacking in pericyte and basement membrane coverage. These features give rise to an inflammatory tumor microenvironment, increased interstitial pressure, and areas of chronic and intermittent hypoxia, driving tumor progression and metastasis. They also contribute to radioresistance and poor therapeutic drug delivery. Strategies to improve tumor vasculature through vascular disruption or antiangiogenic drugs have been used to improve the response to traditional therapies. Antiangiogenic agents have been shown to transiently stabilize abnormal tumor vasculature by restoring the balance of the proangiogenic and antiangiogenic factors inducing a more functional tumor vasculature and improving perfusion, basement membrane structure, and

Research funded by Massey Cancer Center Grant 2P30CA0116059, and the VCU Department of Radiation Oncology Research Funds.

https://doi.org/10.1124/jpet.117.245258. reducing interstitial pressure (Tong et al., 2004; Winkler et al., 2004; Jain, 2005). This process has been called vascular normalization and results in a transient window for improved drug therapy (Qayum et al., 2012; Sorace et al., 2016).

Vasculature normalization has been achieved with agents that inhibit vascular endothelial growth factor (VEGF)-dependent pathways, thereby targeting and pruning the immature vessels of tumors. Evidence from preclinical and clinical studies of anti-VEGF therapy suggests that the efficacy of traditional therapy is improved when administered concurrently. Thus, bevacizumab (Avastin; Genentech, South San Francisco, CA), a monoclonal antibody against VEGF, promotes vascular normalization and, when combined with other cytotoxic agents, shows significant improvement in antitumor efficacy (Miller et al., 2007). Combretastatin, a vascular disrupting agent that targets the tumor vessel endothelium, inhibits VEGF and VEGF receptor 2, resulting in a more normal vessel phenotype (Su et al., 2016). Although promising agents have been identified, the clinical results with these agents have not been as impressive as those obtained in preclinical studies. Many of these antiangiogenic agents are not suitable for long-term use because of the effects on normal endothelium and wound repair. Furthermore, only a transient

ABBREVIATIONS: ANOVA, analysis of variance; $\mathrm{BH}$, dihydrobiopterin; $\mathrm{BH} 4$, tetrahydrobiopterin; eNOS, endothelial nitric oxide synthase; $\% \mathrm{HbO}_{2}$, percentage of oxygenated hemoglobin; HPLC, high-performance liquid chromatography; IR, irradiation; MSOT, multispectral optoacoustic tomography; NO, nitric oxide; NOS, nitric oxide synthase; $\mathrm{O}_{2}^{-}$, superoxide; OCT, optimal cutting temperature; $\alpha$ SMA, $\alpha$-smooth muscle actin; SMC, smooth muscle cell; SP, sepiapterin; VEGF, vascular endothelial growth factor. 
window of efficacy is available because of the development of acquired resistance to these targeted therapies. Thus, improvements in clinical outcomes to this point have been only small delays in tumor relapse (Bergers and Hanahan, 2008).

Much like the inflammatory microenvironment of a tumor, chronic inflammatory diseases such as diabetes and atherosclerosis are associated with an abnormal vasculature and disrupted nitric oxide (NO) signaling of the endothelium. A mechanism that has been well described in the vasculature literature, but that has been largely overlooked in tumor biology, is that NO synthase (NOS) can have two activities: $\mathrm{NO}$ or $\mathrm{O}_{2}^{-} /$peroxynitrite synthase activities. An important determinant of these relative activities is tetrahydrobiopterin (BH4), a necessary cofactor for NOS generation of NO. When $\mathrm{BH} 4$ levels are low, electron transfer in the active site of the enzyme becomes uncoupled from L-arginine oxidation, resulting in the production of $\mathrm{O}_{2}^{-}$in lieu of $\mathrm{NO}$ (Vásquez-Vivar et al., 1998, 2003). Under certain inflammatory conditions, BH4 is limiting as a result of decreased levels of GTP cyclohydrolase I, the rate-limiting enzyme in $\mathrm{BH} 4$ production, or by direct oxidation to dihydrobiopterin (BH2) (Mitchell et al., 2003; Zheng et al., 2003). The ratio of [BH4] to its oxidation product [BH2] is critical because both bind the active site with equal affinity; however, when $\mathrm{BH} 2$ is bound, the production of $\mathrm{O}_{2}^{-}$ predominates (Crabtree et al., 2008).

NO signaling is critical for vascular function, and the uncoupling of endothelial NOS (eNOS) in endothelial cells may partly explain the poor vasculature structure found within solid tumors. Our recent studies have shown in diverse tumor types both in vitro and in vivo that the $\mathrm{BH} 4 / \mathrm{BH} 2$ ratio is 2 -fold to 50-fold higher in normal tissue compared with tumors. In the case of spontaneous MMTV-neu tumors in vivo, the mean $\mathrm{BH} 4 / \mathrm{BH} 2$ ratio is $1.0 \pm 0.2$ compared with $3.8 \pm 0.5$ in the mammary fat pad. The $\mathrm{BH} 4 / \mathrm{BH} 2$ ratio in a human colorectal biopsy sample was 2.3 (range, $1.5-3.5 ; n=4$ ) compared with that of a biopsy sample from adjacent normal tissue of 4.5 (range, $3.5-6.1 ; n=4$ ). Furthermore, in animal tumor models the $\mathrm{BH} 4 / \mathrm{BH} 2$ ratio is increased with the exogenous BH4 metabolic precursor sepiapterin (SP). For example, in the MMTV-neu model, the tumor $\mathrm{BH} 4 / \mathrm{BH} 2$ ratio increases to $2.8 \pm 0.5$ with oral administration of SP. The consequences of therapeutically increasing the $\mathrm{BH} 4 / \mathrm{BH} 2$ ratio is the recoupling NOS activity, leading to enhanced generation of NO and causing a shift from proinflammatory/prosurvival signaling to anti-inflammatory/proapoptotic signaling resulting in the inhibition of spontaneous tumor growth (Rabender et al., 2015).

Previous studies demonstrated that SP-induced NOS recoupling stimulates angiogenesis and functional recovery of dysfunctional endothelium in diabetes, hypertension, and myocardial infarction (Jo et al., 2011; Shimazu et al., 2011; Roe et al., 2013). Having previously demonstrated that NOS can be "recoupled" and NO production can be restored through the treatment of tumor cells or animals with SP, we examined whether SP also normalizes tumor vasculature structure and function, promoting radiosensitivity and improving drug uptake in the MMTV-neu spontaneous breast tumor model.

\section{Materials and Methods}

Reagents. Doxorubicin was from Sigma-Aldrich (St. Louis, MO). L-SP (cat. no. 11.225) was from Schircks Laboratories (Jona,
Switzerland). The Hypoxyprobe-1 Kit was from Hypoxyprobe Inc. (Burlington, MA). Anti-CD31 was from BD Biosciences (Franklin Lakes, NJ). Hoechst 33342 stain was from Life Technologies (Carlsbad, CA). $\alpha$-Smooth muscle actin ( $\alpha$ SMA) was from EMD Millipore (Billerica, MA).

Animals. Breeding pairs of MMTV-neu mice were from The Jackson Laboratory (Bar Harbor, ME). The mice were housed under conditions of 12-hour dark/light cycles and had access to water and food ad libitum. The experiments were conducted under the Guide for the Care and Use of Laboratory Animals published by the National Institutes of Health (revised 2011; Bethesda, MD). The study protocol was approved by the Institutional Animal Care and Use Committee of Virginia Commonwealth University.

Optoacoustic Imaging. In multispectral optoacoustic tomography (MSOT), tissues are excited by multiple-wavelength illumination, and, in response to light absorption, thermoelastic materials (like hemoglobin) produce ultrasonic waves. MSOT imaging was performed using an MSOT inVision 256-TF small animal scanner (iThera Medical GmbH, Munich, Germany). The laser system provides excitation pulses with a duration of 9 nanoseconds at wavelengths from 680 to $980 \mathrm{~nm}$ at a repetition rate of $10 \mathrm{~Hz}$. For defining the oxyhemoglobin and deoxyhemoglobin signals, a minimum of the following wavelengths were used in all acquisitions: 700, 730, 760, 800,860 , and $900 \mathrm{~nm}$.

Mice are submerged in a water tank in a horizontal position in a holder and are wrapped in a thin polyethylene membrane to prohibit direct contact between water and mouse but still allow for acoustic coupling. Isoflurane anesthesia and oxygen are supplied through a breathing mask. The mouse and holder can be translated using a linear stage to enable imaging of multiple transverse slices. The resolution of the translational stage was $0.5 \mathrm{~mm}$.

To create an image at one wavelength, signals from 20 subsequent excitation pulses are averaged to compensate for laser pulse fluctuations and animal motion as well as to improve the signal-to-noise ratio. Images are reconstructed using a standard back-projection algorithm, and three-dimensional images are reconstructed using the interpolated model-matrix inversion. Both are applied from within the ViewMSOT software suite supplied with the iThera Medical inVision 256-TF small animal scanner system. After image reconstruction, linear spectral unmixing is applied to detect and separate oxyhemoglobin and deoxyhemoglobin signals from other photoabsorbing tissue elements. For each pixel in the image, the method fits the total measured optoacoustic spectrum to the known absorption spectra of oxyhemoglobin and deoxyhemoglobin. This produces individual component images, each visualizing the biodistribution of the respective absorber. The oxyhemoglobin and deoxyhemoglobin signal intensities in the tumors are calculated by drawing regions of interest over the tumors and measuring the respective signal intensities within those regions of interest.

Immunofluorescence. For hypoxic area studies, the hypoxia marker pimonidazole $(70 \mathrm{mg} / \mathrm{kg}$, i.p.) and the perfusion marker Hoechst 33342 (1 mg/mouse, i.v.; Sigma-Aldrich) are administered 1 hour and 1 minute prior to animal sacrifice and tumor excision, respectively. Tumors are embedded in optimal cutting temperature (OCT) compound and stored at $-80^{\circ} \mathrm{C}$ until processing. Cryosections $(8 \mu \mathrm{m})$ from the middle of the tumor are fixed in $4 \%$ paraformaldehyde, permeabilized, and blocked with goat serum prior to staining with the following antibodies: anti-CD31 (BD Biosciences), anti-SMA (EMD Millipore), and anti-pimonidazole (Hypoxyprobe Inc.). The samples are subsequently washed and incubated for 1 hour at room temperature with the appropriate dye-conjugated Alexa Fluor 594 and Alexa Fluor 488 secondary antibodies (Invitrogen, Carlsbad, CA). Images are captured using the Ariol Digital Pathology Platform (Leica Biosystems, Wetzlar, Germany) and quantified with ImageJ using DAPI (4',6'-diamidino-2-phenylindole dihydrochloride) staining for normalization. An advantage to using the Ariol Digital Pathology Platform is the ability of the microscope to stitch together many images to capture the entire tissue section; however, to facilitate 
visualization of the staining for some images, only representative sections of the tissue are shown. In all experiments the statistical analysis has been performed on the whole section.

Staining for apoptosis is accomplished using the ApopTag Plus Fluorescein In Situ Apoptosis Detection Kit (cat. no. S7111; EMD Millipore).

Doxorubicin Analysis in Tissues. Mice were anesthetized with isoflurane prior to cervical dislocation. Tumors were removed and snap frozen in liquid nitrogen prior to analysis. The method for analysis of doxorubicin levels in tissue is adapted from Al-Abd et al. (2009) and Qayum et al. (2012). Doxorubicin levels were measured by high-performance liquid chromatography (HPLC; Dionex Ultimate 300; Thermo Fisher Scientific, Waltham, MA), using a Waters (Milford, MA) Spherisorb $5 \mu \mathrm{M}$ ODS1 column with fluorescence detection at excitation and emission wavelengths of 480 and $550 \mathrm{~nm}$, respectively.

Radiotherapy. Computed tomography-based treatment planning and execution used the Small Animal Radiation Research Platform from Xstrahl (Suwanee, GA). Animals were anesthetized with $2 \%$ isoflurane during computed tomography scanning and radiotherapy. A single dose of 4-Gy irradiation (IR) was delivered, and 24 hours later animals were sacrificed and excised tumors were frozen in OCT compound for future analysis.

Statistical Analysis. Except where indicated, a one-way ANOVA with Dunnett's post hoc test was used for statistical analysis, with $P<0.05$ considered to be statistically significant. A two-way ANOVA was used to assess the impact of drug interactions (Slinker, 1998).

\section{Results}

Effects of SP on Hypoxia and Tumor Blood Flow in the MMTV-Neu Spontaneous Breast Tumor Model. To evaluate vascular function in these tumors we used MSOT to measure the percentage of oxygenated hemoglobin $\left(\% \mathrm{HbO}_{2}\right)$ in the tumors. Mice bearing tumors approximately $500 \mathrm{~mm}^{3}$ in size were treated with $1 \mathrm{mg} / \mathrm{kg}$ per day SP by oral gavage for 6 days to improve NOS coupling. MSOT was performed on day 0 , day 5 , day 8 , and day 16 . With the SP-treated animals, the MMTV tumors displayed an increased $\% \mathrm{HbO}_{2}$ with a significant increase after 6 days of SP treatment, with maximum effect measured at 8 days post-treatment. Figure 1 shows that the increase in oxygenation, relative to untreated controls, was $30 \%(P<0.01)$. A significant improvement in tumor oxygenation was sustained for the duration of the experiment. In contrast, there was a progressive decrease in $\% \mathrm{HbO}_{2}$ in control MMTV tumors of untreated animals during this same time period. This was not unexpected since hypoxia increases over time with tumor progression, though the results were not significant $(P=0.08)$. These results with a spontaneous tumor model provide evidence that SP-treated tumors are not outgrowing their vasculature in contrast to control tumors and as a result are better oxygenated.

The increased $\% \mathrm{HbO}_{2}$ due to SP, if due to vascular normalization, should also be observed as a reduction in hypoxia. To confirm a reduction in hypoxia, we treated another group of animals with SP $1 \mathrm{mg} / \mathrm{kg}$ per day for 6 days and on day 8 injected pimonidazole, a marker for tumor hypoxia, intraperitoneally 60 minutes prior to sacrifice and Hoechst stain, a marker for tumor perfusion, intravenously 1 minute prior to sacrifice. Cross sections of tumors were analyzed by immunofluorescence, which confirmed the reduction in tumor hypoxia and increased perfusion. Figure 2 shows that SP-treated animals showed an almost $40 \%$

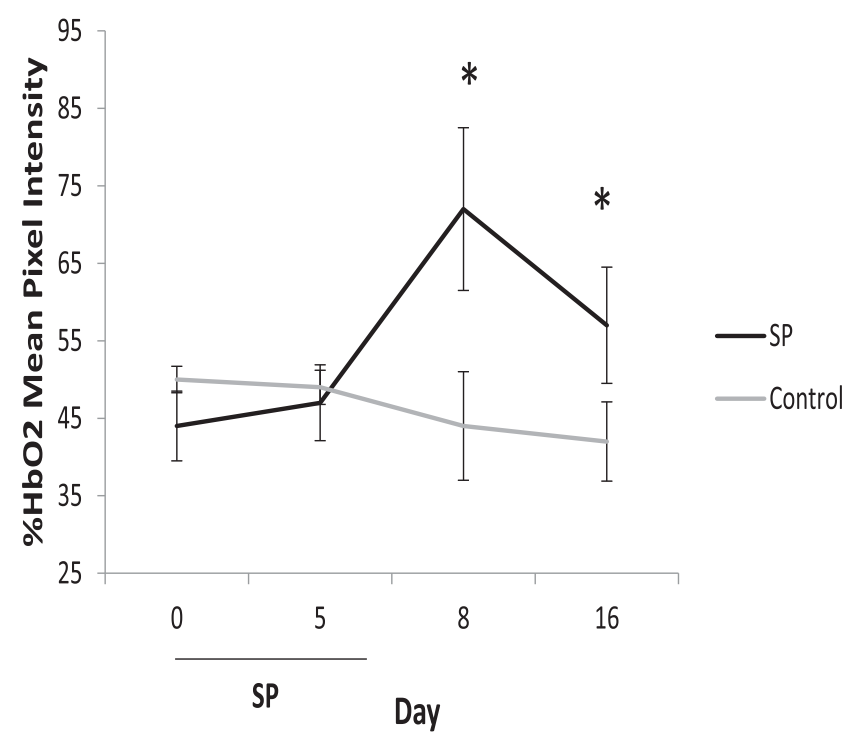

Fig. 1. Effect of SP treatment on oxygenated hemoglobin levels in MMTV spontaneous tumors. Mice were treated with $1 \mathrm{mg} / \mathrm{kg}$ per day SP for 6 days by oral gavage. $\% \mathrm{HbO}_{2}$ was measured by MSOT on day 0 before treatment began, and on days 5,8 , and 16 . SP increased the $\% \mathrm{HbO}_{2}$ by $30 \%$ at day 8 . ${ }^{*} P<0.01$ between SP and control; $n=7$ tumors for both groups.

reduction in pimonidazole-Hoechst staining over control $(P<0.05)$.

SP Normalizes the Structure of Tumor Vasculature. Tumor angiogenesis is characterized by an immature vasculature lacking in the recruitment of smooth muscle cells (SMCs) to and coverage of endothelial cells. Loss of SMC coverage contributes greatly to the hemorrhagic vessels seen in tumors. We examined whether SP treatment increased SMC recruitment to MMTV-neu tumor vasculature by measuring $\alpha$ SMA staining. Mice were given $1 \mathrm{mg} / \mathrm{kg}$ per day SP or vehicle for 6 days by oral gavage. On day 8 , the animals were sacrificed and tumors were embedded in OCT compound. Immunofluorescent analysis of $\alpha$ SMA shows a 3 -fold increase in staining in SP treated tumors compared with vehicle animals $(P<0.001)$ (Fig. 3A).

We also demonstrate an average $30 \%$ decrease in overall CD31 staining arising from decreasing vessel density (Fig. 3B). Costaining of CD31 and $\alpha$ SMA is used as an indicator of the attachment of mural cells to endothelial cells. Vehicle-treated tumors stained for CD31, but with very little associated $\alpha$ SMA; however, in tumors that had been treated with SP, $\alpha$ SMA costaining increased by more than 2 -fold over vehicle-treated mice, all of which was associated with CD31positive cells (Fig. 4).

Improved Tumor Oxygenation Correlates with Increased Radiation-Induced Tumor Cell Apoptosis. Hypoxia by multiple mechanisms promotes radioresistance (Vaupel, 2004). Furthermore, it is well known that the presence of molecular oxygen improves tumor cell killing by radiation through the increased presence of free radicals that "fix" DNA damage. We tested whether the normalization of tumor vasculature by SP would translate to more effective tumor cell killing by radiation when measured by apoptosis. Mice were given $1 \mathrm{mg} / \mathrm{kg}$ per day SP or vehicle for 6 days. On day 8 , corresponding to the peak $\% \mathrm{HbO}_{2}$ levels, we administered a dose of 4 Gy to each tumor using the Small Animal Radiation Research Platform (Xstrahl). The tumor was excised 


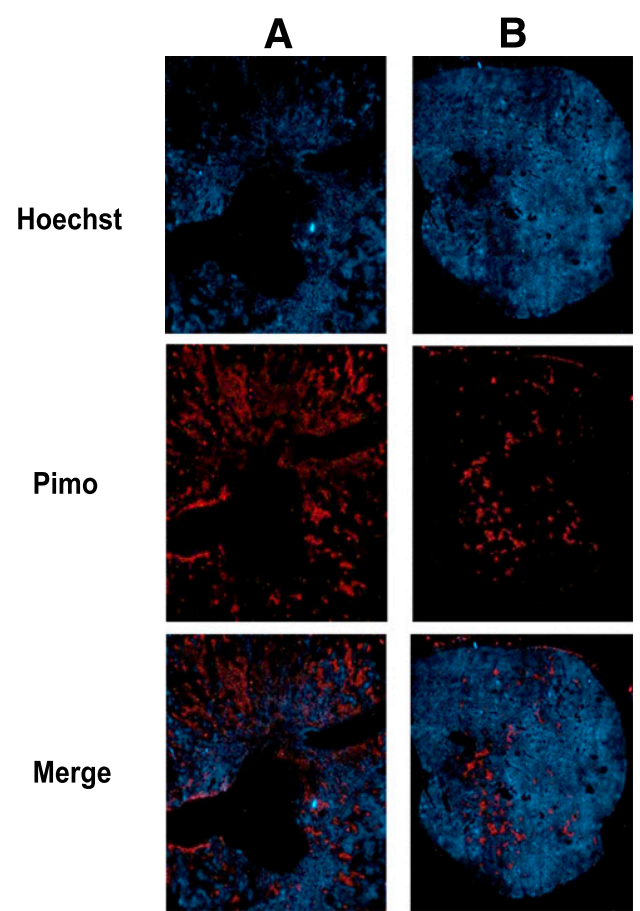

24 hours post- radiation exposure for analysis. Figure 5 shows that whereas both SP and radiation alone showed an increase in tumor cell apoptosis, when animals were given SP before radiation there was a greater than 2 -fold increase in tumor cell killing measured by apoptosis over radiation plus vehicle $(P<$ 0.01). Using two-way ANOVA, we were able to demonstrate a synergistic effect for the combination of SP and IR over SP and IR alone (Slinker, 1998) $(P=0.045)$.

Pretreatment with SP Enhances Tumor Uptake of Doxorubicin and Increases Tumor Cell Apoptosis. Previous studies demonstrated that the normalization of tumor vasculature allows for more efficient delivery of chemotherapeutic agents (Tong et al., 2004; Dings et al., 2007; Qayum et al., 2012). To determine how SP-induced changes in vasculature impact the distribution of chemotherapeutic agents in the tumor, we gave a single intraperitoneal injection of vehicle or $30 \mathrm{mg} / \mathrm{kg}$ doxorubicin to mice that had been treated with vehicle or $1 \mathrm{mg} / \mathrm{kg}$ per day SP by oral gavage for 6 days. Based on studies by Primeau et al. (2005) and Qayum et al. (2012), we used a high dose of doxorubicin, $30 \mathrm{mg} / \mathrm{kg}$, to enhance detection capabilities. At 60 minutes after doxorubicin injection, animals were sacrificed, tumors were removed,
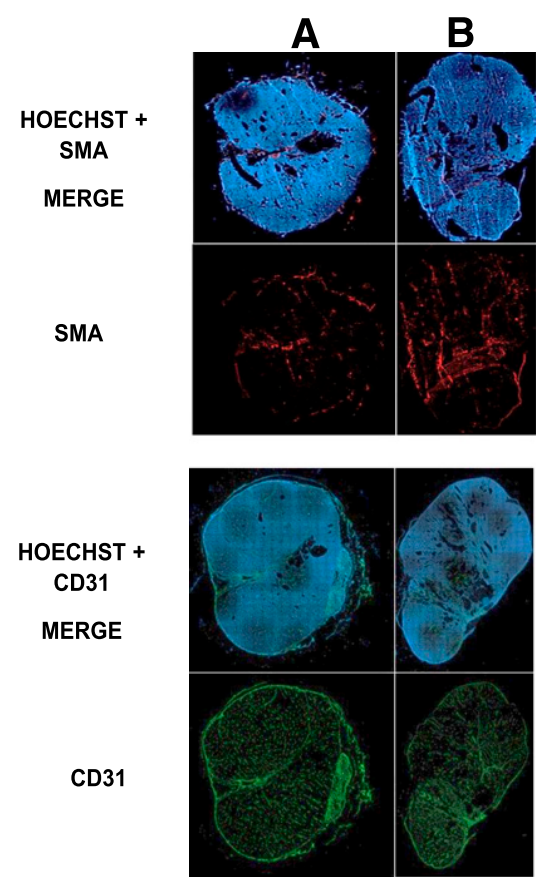
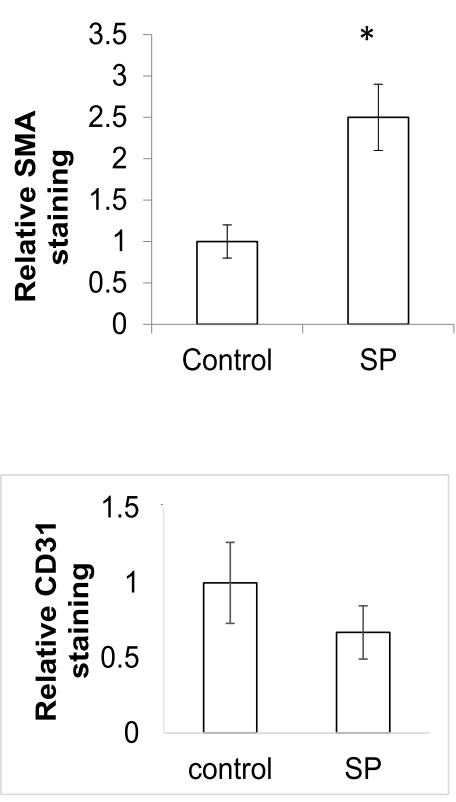

Fig. 3. SP improves tumor vasculature. (A) Control mice. (B) SP-treated mice. Mice treated with $1 \mathrm{mg} / \mathrm{kg}$ SP orally for 6 days had an almost 3 -fold increase in SMA staining (red) at 8 days after the beginning of treatment. (B) CD31 staining (green) in MMTV tumors treated with SP decreased a little more than $30 \%$ vs. CD31 staining in control tumors. (I) SMA quantification. (II) CD31 quantification. $N=5$ mice and $n=6$ tumors for each group. $* P<0.001$ by paired Student's $t$ test. 

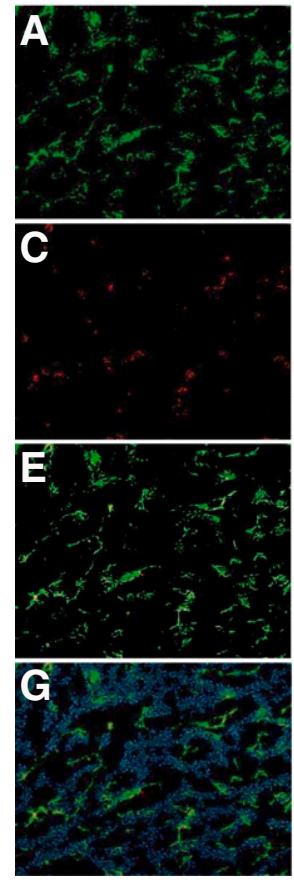
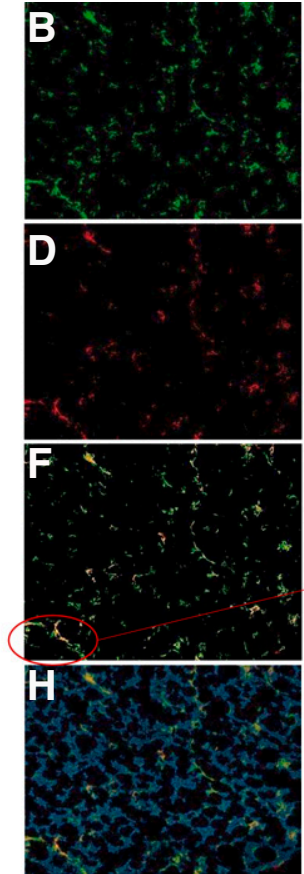

I.

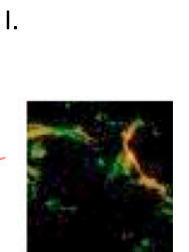

II.

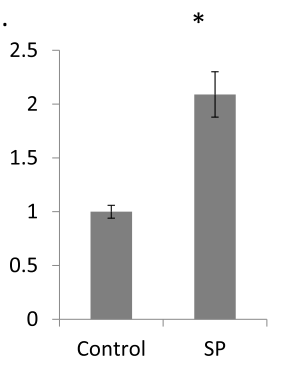

Fig. 4. SP recruits mural cells, normalizing tumor vasculature. Mice were treated with vehicle or $1 \mathrm{mg} / \mathrm{kg}$ per day SP for 6 days by oral gavage. On day 8 , the tumors were removed and analyzed by immunofluorescence for costaining of CD31 and SMA. (A, C, E, and G) Vehicle-treated mice. (B, D, F, and H) SP-treated mice. (A and B) CD31 (green). (C and D) SMA (red). (E and F) Merged image of CD31 and SMA. (G and H) Merged image of Hoechst stain, CD31, and SMA. Orange staining is indicative of pericyte coverage of endothelial cells. (I) Zoom. (II) Quantification of SMA/CD31 ratio, normalized to control. $N=5$ mice per group, $n=7$ tumors for control, and $n=8$ for SP. ${ }^{*} P<0.01$. and extracted for HPLC analysis of doxorubicin. There was almost a 2-fold increase in tumor-associated doxorubicin in those animals receiving $\mathrm{SP}(2.28$ and $1.3 \mu \mathrm{mol} / 1$, respectively; $P<0.05$ ) (Fig. 6). The impact of this increased tumor doxorubicin on apoptosis is shown in Fig. 7. Mice were treated with vehicle or $8 \mathrm{mg} / \mathrm{kg}$ doxorubicin intraperitoneally for 1 day, after which the tumors were removed and analyzed by immunofluorescence for apoptosis. Monotherapy with doxorubicin caused an increase in the levels of apoptosis compared with vehicle-treated animals; however, when pretreated with SP, there was a synergistic effect of combined therapies with an almost 3 -fold increase in apoptotic cells compared with doxorubicin alone $(P<0.01$ by two-way ANOVA).

\section{Discussion}

Based on the criteria of enhanced $\% \mathrm{HbO}_{2}$, as measured by photoacoustic imaging, reduced staining with the hypoxia probe pimonidazole, and both increased SMC staining and endothelial coverage, SP normalizes the vasculature of MMTV-neu tumors. These changes in tumor vasculature increased the sensitivity of these tumors to ionizing radiation while also enhancing the distribution of doxorubicin within the tumor, leading to more effective tumor cell killing.

Having previously demonstrated that SP increases the $\mathrm{BH} 4 / \mathrm{BH} 2$ ratio recoupling NOS activity in the MMTV-neu tumor model, one mechanistic explanation for these findings is that $\mathrm{SP}$, by enhancing the $\mathrm{BH} 4 / \mathrm{BH} 2$ ratio, recouples NOS generating NO. Recoupling of NOS activity is aligned with several studies suggesting that modulation of NO signaling may be a novel strategy for controlling tumor vessel structure and function. NOS activity impacting tumor vasculature has been demonstrated by Kashiwagi et al. (2008), who showed that the inhibition of NOS in U87MG gliomas led to improved oxygen delivery and a more normal phenotype. Recoupling of NOS by SP will have many of the same downstream effects as inhibiting the activity of uncoupled NOS, with the addition of the generation of low levels of NO. Low levels of NO have been demonstrated to preserve physiologic blood vessel integrity (Fukumura et al., 2006). Angiogenesis defects in Akt1-null mice can be rescued by the expression of a constitutively active eNOS, a major target of Akt (Schleicher et al., 2009). Previous studies also showed that endothelial cell-derived NO induces the migration of mural cell precursors toward endothelial cells and, using eNOS ${ }^{-1-}$ mice, showed that eNOS-derived NO is the main promoter of perivascular recruitment. In the B16M model, a decrease in perivascular cell (pericytes and SMCs) coverage was found in cells with low NOS expression (Kashiwagi et al., 2005).

The first antiangiogenic agents, such as bevacizumab or the phosphoinositide 3-kinase inhibitor pictilisib, targeted one specific protein or a specific pathway. More recent antiangiogenic agents, multitarget tyrosine kinase inhibitors such as sorafenib or nintedanib, inhibit several proangiogenic factors (Scagliotti and Govindan, 2010; Liang et al., 2014). Regardless of the specific mechanism of action, they are limited by normal tissue toxicity because in most cases they target wound repair mechanisms. For this reason, they are also ultimately rendered ineffective by cellular adaptation due to the multiplicity of wound repair mechanisms (Bergers and Hanahan, 2008). SP also targets a single pathway, but the end product, NO, has multiple effects. As discussed above, it promotes perivascular recruitment but also regulates VEGF activity (Dulak et al., 2000 ), reduces infiltration of inflammatory macrophages, and reduces inflammation as measured by interleukin- 6 and interleukin-1 $\beta$ (Cardnell et al., 2013). Tumor-associated macrophages are known to be important for tumor progression as well, and SP may influence the function of these macrophages in normalizing vasculature. Fibroblast growth factor 2, 

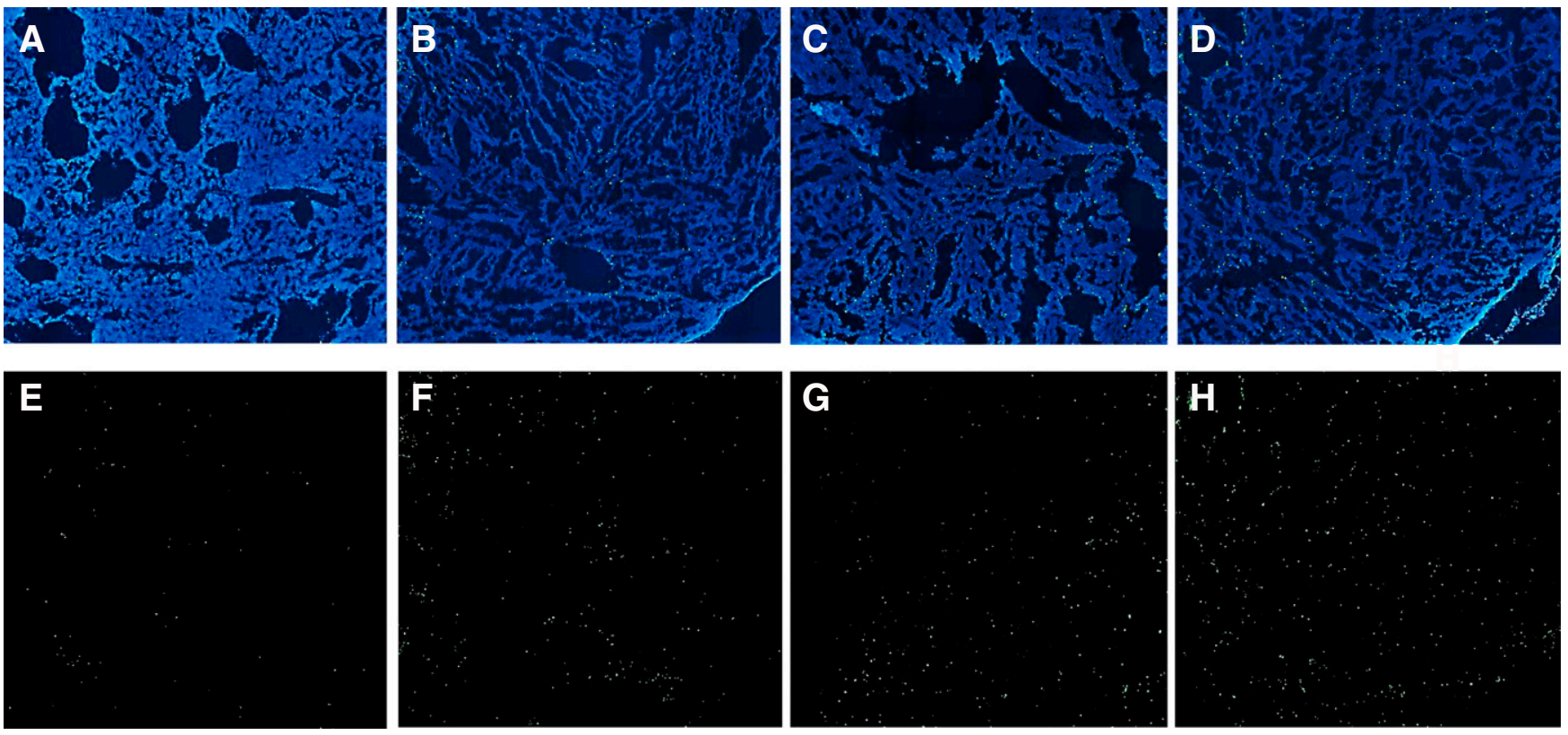

I.

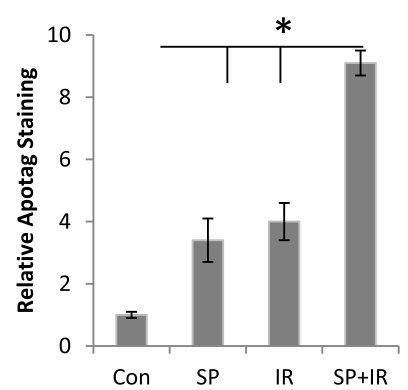

Fig. 5. Tumor oxygenation enhances IR-induced cell killing. Mice were treated with vehicle or $1 \mathrm{mg} / \mathrm{kg}$ per day SP for 6 days by oral gavage. On day 8 , the IR and SP+IR groups were given one 4-Gy dose. Twenty-four hours later, all animals were euthanized and the tumors were removed. Apoptosis was imaged using an EMD Millipore Apoptag In Situ Fluorescein Apoptosis Detection Kit. (A-D) Hoechst + Apoptag. (E-H) Apoptag. Images are of mice given vehicle (A and E), SP only (B and F), 4-Gy IR (C and G), and SP+4-Gy IR (D and H). $N=5$ mice for control (Con) and SP, seven mice for IR and SP +IR. $n=6$ tumors for control, $n=6$ tumors for SP, $n=10$ tumors for IR, and $n=10$ tumors for SP+IR. (I) Quantification. $* P<0.01$.

transforming growth factor- $\beta$, and angiopoietin 1/angiopoietin 2 are also potentially involved, and all are modified by NO-dependent pathways or inflammation. Normal tissue vasculature, on the other hand, should be minimally effected by $\mathrm{SP}$ treatment since the $\mathrm{BH} 4 / \mathrm{BH} 2$ ratio in these tissues is normally tightly regulated through positive and negative feedback mechanisms (Werner et al., 2011).

The majority of published studies also show a significant decrease in CD31 density as a hallmark of tumor normalization; however, our results, although showing a trend of decreased CD31 density, did not reach significance. An advantage to using the Ariol Digital Pathology Platform (Leica Biosystems) to visualize the tumor sections is that it takes a series of images and stitches them together allowing visualization of the entire tumor section. This facilitates quantification and comparisons over the whole tumor, not just in small windows. We found this to be important in a study of this nature as we could find many areas in which tumor vessel density had decreased, but we also came across other areas that were still very dense. Although there was an overall 30\% decrease in CD31 staining, this decrease failed to reach significance $(P=0.06)$ because of the pockets of dense CD31 staining. Adjusting treatment times, doses, and duration of SP exposure may further enhance tumor vasculature normalization enabling doxorubicin and irradiation to have a more complete impact on the tumor.
Despite the antitumor effectiveness of doxorubicin in the clinic, its use is limited because of the associated cardiotoxicities and myelosuppression that arise after treatment. Tumor vascular normalization with SP may mitigate these toxicities in two ways. Improving delivery to the tumor may

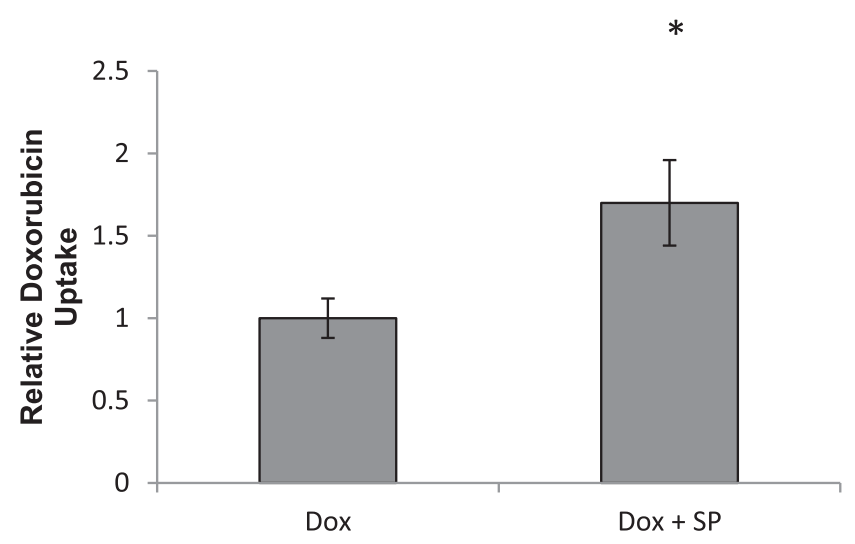

Fig. 6. The effect of SP pretreatment on tumor doxorubicin uptake. The animals were treated with $1 \mathrm{mg} / \mathrm{kg}$ per day SP for 6 days. On day 8 , the mice were injected with vehicle or doxorubicin (Dox) $30 \mathrm{mg} / \mathrm{kg}$ i.v. and sacrificed 60 minutes postinjection. The concentration of doxorubicin was measured by HPLC. $N=4$ mice, $n=7$ tumors for Dox + SP and six tumors for Dox. $* P<0.01$ by paired Student's $t$ test. 

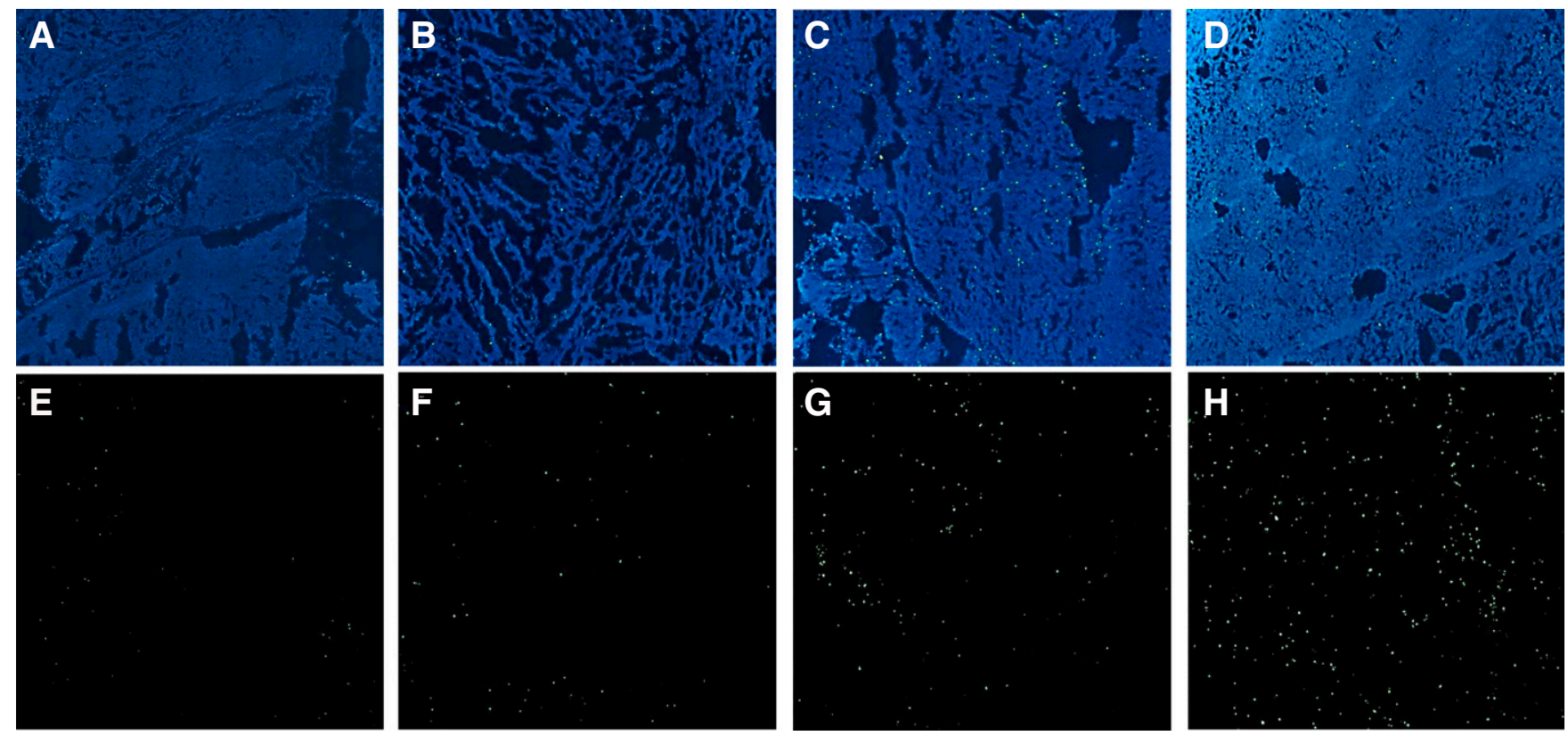

I.

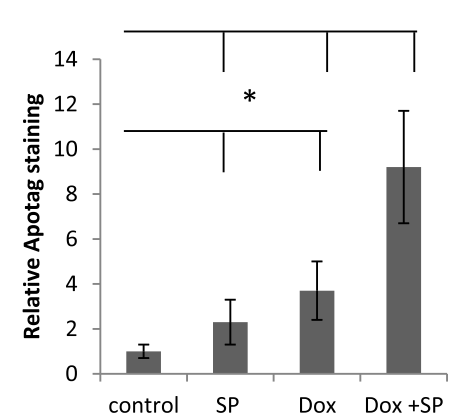

Fig. 7. Doxorubicin (Dox)-induced apoptosis is markedly increased by pretreatment with SP. SP was administered at $1 \mathrm{mg} / \mathrm{kg}$ for 6 days by oral gavage. On day 8, mice were injected with Dox $8 \mathrm{mg} / \mathrm{kg}$ i.p. and euthanized 24 hours later. (A-D) Hoechst + Apoptag. (E-H) Apoptag. Images are control mice (A and E), SP only (B and F), Dox only (C and G), and SP+Dox (D and H). $N=3$ tumors for control, $n=5$ tumors for SP, $n=6$ tumors for Dox + SP, and $n=6$ tumors for Dox. $* P<0.05 ; * P<0.01$.

permit lower doses of doxorubicin to be used for tumor control. In addition to being able to lower the dose of doxorubicin, SP has also been shown to improve cardiac function and reduce fibrosis in models of cardiac ischemia and pressure overload as well as ameliorate diabetic cardiomyopathy (Baumgardt et al., 2016). In addition to low toxicity, SP also demonstrates its own antitumor properties with MMTV-neu and other tumor models (Rabender et al., 2015).

One drawback of using antiangiogenic agents or vascular disruption agents for vasculature normalization is that the effect is transient. Indeed, our previous studies (Cardnell and Mikkelsen, 2011) have demonstrated that the treatment of mice with the NOS inhibitor LNNA (L-NG-nitroarginine) reduced tumor blood flow, resulting in delayed tumor growth and improved animal survival, but ultimately the effect was lost and so was the impact on tumor control. The effect we observed with a 6-day treatment of SP, however, was a continuing significant improvement in tumor oxygenation measured 10 days after the final SP treatment. We are unsure whether the effect of SP on tumor vasculature and oxygenation would be more robust and sustained had we treated the mice for a longer period of time. Future studies will examine longer durations of treatment as well as pretreatment and coadministration of SP with additional chemotherapies. That $\mathrm{BH} 4$ is already used clinically in the long-term treatment of some forms of phenylketonuria (Muntau et al., 2002) and over extended periods of time suggests that the continued administration and examination of the effects on tumor vasculature are potentially viable approaches.

This investigation used SP instead of BH4 because SP is more permeable to cells (Ohashi et al., 2016). SP has not been used as extensively in the clinic as BH4, with only one reported study on its use in the treatment of atypical phenylketonuria due to BH4 deficiency (Curtius et al., 1979). However, in animal studies SP, like BH4, has been effective in treating a number of vascular disorders (e.g., cardiomyopathy and cardiac ischemia/reperfusion injury) (Okazaki et al., 2011; Baumgardt et al., 2016). BH4 is in clinical trials for the treatment of endothelial dysfunction in a number of vascular diseases. Since the $\mathrm{BH} 4 / \mathrm{BH} 2$ ratio of normal tissues is high and the NOS activity of these cells is coupled, BH4 or SP should have minimal effects on normal tissue and vasculature and is therefore a potentially safe alternative to other normalizing agents, such as avastin, which are associated with considerable toxicity (Brandes et al., 2015). Taken together, these findings show great promise for the use of SP 
as a vascular normalizing agent, and further investigation into NOS uncoupling and tumor vasculature is warranted.

\section{Authorship Contributions}

Participated in research design: Rabender, Bruno, Alam, Sundaresan, Zweit, Mikkelsen.

Conducted experiments: Rabender, Bruno, Alam, Sundaresan.

Performed data analysis: Rabender, Bruno, Sundaresan.

Wrote or contributed to the writing of the manuscript: Rabender, Bruno, Mikkelsen.

\section{References}

Al-Abd AM, Kim NH, Song SC, Lee SJ, and Kuh HJ (2009) A simple HPLC method for doxorubicin in plasma and tissues of nude mice. Arch Pharm Res 32:605-611. Baumgardt SL, Paterson M, Leucker TM, Fang J, Zhang DX, Bosnjak ZJ, Warltier DC, Kersten JR, and Ge ZD (2016) Chronic co-administration of sepiapterin and L-citrulline ameliorates diabetic cardiomyopathy and myocardial ischemia/ reperfusion injury in obese type 2 diabetic mice. Circ Heart Fail 9:e002424.

Bergers G and Hanahan D (2008) Modes of resistance to anti-angiogenic therapy. Nat Rev Cancer 8:592-603.

Brandes AA, Bartolotti M, Tosoni A, Poggi R, and Franceschi E (2015) Practical management of bevacizumab-related toxicities in glioblastoma. Oncologist 20:166-175.

Cardnell RJ and Mikkelsen RB (2011) Nitric oxide synthase inhibition enhances the antitumor effect of radiation in the treatment of squamous carcinoma xenografts. PLoS One 6:e20147.

Cardnell RJ, Rabender CS, Ross GR, Guo C, Howlett EL, Alam A, Wang XY, Akbaral HI, and Mikkelsen RB (2013) Sepiapterin ameliorates chemically induced murine colitis and azoxymethane-induced colon cancer. J Pharmacol Exp Ther 347:117-125.

Carmeliet P and Jain RK (2011) Principles and mechanisms of vessel normalization for cancer and other angiogenic diseases. Nat Rev Drug Discov 10:417-427.

Crabtree MJ, Smith CL, Lam G, Goligorsky MS, and Gross SS (2008) Ratio of 5,6, 7,8-tetrahydrobiopterin to 7,8-dihydrobiopterin in endothelial cells determines glucose-elicited changes in NO vs. superoxide production by eNOS. Am J Physiol Heart Circ Physiol 294:H1530-H1540.

Curtius HC, Niederwieser A, Viscontini M, Otten A, Schaub J, Scheibenreiter S, and Schmidt H (1979) Atypical phenylketonuria due to tetrahydrobiopterin deficiency. Diagnosis and treatment with tetrahydrobiopterin, dihydrobiopterin and sepiapterin. Clin Chim Acta 93:251-262.

Dings RP, Loren M, Heun H, McNiel E, Griffioen AW, Mayo KH, and Griffin RJ (2007) Scheduling of radiation with angiogenesis inhibitors anginex and avastin improves therapeutic outcome via vessel normalization. Clin Cancer Res $\mathbf{1 3}$ 3395-3402

Dulak J, Józkowicz A, Dembinska-Kiec A, Guevara I, Zdzienicka A, ZmudzinskaGrochot D, Florek I, Wójtowicz A, Szuba A, and Cooke JP (2000) Nitric oxide induces the synthesis of vascular endothelial growth factor by rat vascular smooth muscle cells. Arterioscler Thromb Vasc Biol 20:659-666.

Fukumura D, Kashiwagi S, and Jain RK (2006) The role of nitric oxide in tumour progression. Nat Rev Cancer 6:521-534.

Jain RK (2005) Normalization of tumor vasculature: an emerging concept in antiangiogenic therapy. Science 307:58-62.

Jo H, Otani H, Jo F, Shimazu T, Okazaki T, Yoshioka K, Fujita M, Kosaki A, and Iwasaka T (2011) Inhibition of nitric oxide synthase uncoupling by sepiapterin improves left ventricular function in streptozotocin-induced diabetic mice. Clin Exp Pharmacol Physiol 38:485-493.

Kashiwagi S, Izumi Y, Gohongi T, Demou ZN, Xu L, Huang PL, Buerk DG, Munn LL, Jain RK, and Fukumura D (2005) NO mediates mural cell recruitment and vessel morphogenesis in murine melanomas and tissue-engineered blood vessels. J Clin Invest 115:1816-1827.

Kashiwagi S, Tsukada K, Xu L, Miyazaki J, Kozin SV, Tyrrell JA, Sessa WC, Gerweck LE, Jain RK, and Fukumura D (2008) Perivascular nitric oxide gradients normalize tumor vasculature. Nat Med 14:255-257.

Liang W, Wu X, Hong S, Zhang Y, Kang S, Fang W, Qin T, Huang Y, Zhao H, and Zhang L (2014) Multi-targeted antiangiogenic tyrosine kinase inhibitors in advanced non-small cell lung cancer: meta-analyses of 20 randomized controlled trials and subgroup analyses. PLoS One 9:e109757.

Miller K, Wang M, Gralow J, Dickler M, Cobleigh M, Perez EA, Shenkier T, Cella D, and Davidson NE (2007) Paclitaxel plus bevacizumab versus paclitaxel alone for metastatic breast cancer. N Engl J Med 357:2666-2676.
Mitchell BM, Dorrance AM, and Webb RC (2003) GTP cyclohydrolase 1 downregulation contributes to glucocorticoid hypertension in rats. Hypertension 41: $669-674$.

Muntau AC, Röschinger W, Habich M, Demmelmair H, Hoffmann B, Sommerhoff CP, and Roscher AA (2002) Tetrahydrobiopterin as an alternative treatment for mild phenylketonuria. $N$ Engl J Med 347:2122-2132.

Ohashi A, Saeki Y, Harada T, Naito M, Takahashi T, Aizawa S, and Hasegawa H (2016) Tetrahydrobiopterin supplementation: elevation of tissue biopterin levels accompanied by a relative increase in dihydrobiopterin in the blood and the role of probenecid-sensitive uptake in scavenging dihydrobiopterin in the liver and kidney of rats. PLoS One 11:e0164305.

Okazaki T, Otani H, Shimazu T, Yoshioka K, Fujita M, and Iwasaka T (2011) Ascorbic acid and N-acetyl cysteine prevent uncoupling of nitric oxide synthase and increase tolerance to ischemia/reperfusion injury in diabetic rat heart. Free Radic Res 45:1173-1183.

Primeau AJ, Rendon A, Hedley D, Lilge L, and Tannock IF (2005) The distribution of the anticancer drug doxorubicin in relation to blood vessels in solid tumors. Clin Cancer Res 11:8782-8788.

Qayum N, Im J, Stratford MR, Bernhard EJ, McKenna WG, and Muschel RJ (2012) Modulation of the tumor microvasculature by phosphoinositide-3 kinase inhibition increases doxorubicin delivery in vivo. Clin Cancer Res 18:161-169.

Rabender CS, Alam A, Sundaresan G, Cardnell RJ, Yakovlev VA, Mukhopadhyay ND, Graves P, Zweit J, and Mikkelsen RB (2015) The role of nitric oxide synthase uncoupling in tumor progression. Mol Cancer Res 13:1034-1043.

Roe ND, He EY, Wu Z, and Ren J (2013) Folic acid reverses nitric oxide synthase uncoupling and prevents cardiac dysfunction in insulin resistance: role of $\mathrm{Ca} 2+/$ calmodulin-activated protein kinase II. Free Radic Biol Med 65:234-243.

Scagliotti G and Govindan R (2010) Targeting angiogenesis with multitargeted tyrosine kinase inhibitors in the treatment of non-small cell lung cancer. Oncologist 15:436-446.

Schleicher M, Yu J, Murata T, Derakhshan B, Atochin D, Qian L, Kashiwagi S, Di Lorenzo A, Harrison KD, Huang PL, et al. (2009) The Akt1-eNOS axis illustrates the specificity of kinase-substrate relationships in vivo. Sci Signal 2:ra41.

Shimazu T, Otani H, Yoshioka K, Fujita M, Okazaki T, and Iwasaka T (2011) Sepiapterin enhances angiogenesis and functional recovery in mice after myocardial infarction. Am J Physiol Heart Circ Physiol 301:H2061-H2072

Slinker BK (1998) The statistics of synergism. J Mol Cell Cardiol 30:723-731.

Sorace AG, Quarles CC, Whisenant JG, Hanker AB, McIntyre JO, Sanchez VM, and Yankeelov TE (2016) Trastuzumab improves tumor perfusion and vascular delivery of cytotoxic therapy in a murine model of HER2+ breast cancer: preliminary results. Breast Cancer Res Treat 155:273-284

Su M, Huang J, Liu S, Xiao Y, Qin X, Liu J, Pi C, Luo T, Li J, Chen X, et al. (2016) The anti-angiogenic effect and novel mechanisms of action of Combretastatin A-4. Sci Rep 6:28139.

Tong RT, Boucher Y, Kozin SV, Winkler F, Hicklin DJ, and Jain RK (2004) Vascular normalization by vascular endothelial growth factor receptor 2 blockade induces a pressure gradient across the vasculature and improves drug penetration in tumors. Cancer Res 64:3731-3736.

Vásquez-Vivar J, Kalyanaraman B, and Martásek P (2003) The role of tetrahydrobiopterin in superoxide generation from eNOS: enzymology and physiological implications. Free Radic Res 37:121-127.

Vásquez-Vivar J, Kalyanaraman B, Martásek P, Hogg N, Masters BS, Karoui H, Tordo P, and Pritchard KA, Jr (1998) Superoxide generation by endothelial nitric oxide synthase: the influence of cofactors. Proc Natl Acad Sci USA 95:9220-9225.

Vaupel P (2004) Tumor microenvironmental physiology and its implications for radiation oncology. Semin Radiat Oncol 14:198-206.

Werner ER, Blau N, and Thöny B (2011) Tetrahydrobiopterin: biochemistry and pathophysiology. Biochem $J$ 438:397-414.

Winkler F, Kozin SV, Tong RT, Chae SS, Booth MF, Garkavtsev I, Xu L, Hicklin DJ, Fukumura D, di Tomaso E, et al. (2004) Kinetics of vascular normalization by VEGFR2 blockade governs brain tumor response to radiation: role of oxygenation, angiopoietin-1, and matrix metalloproteinases. Cancer Cell 6:553-563.

Zheng JS, Yang XQ, Lookingland KJ, Fink GD, Hesslinger C, Kapatos G, Kovesdi I, and Chen $\mathrm{AF}$ (2003) Gene transfer of human guanosine 5'-triphosphate cyclohydrolase I restores vascular tetrahydrobiopterin level and endothelial function in low renin hypertension. Circulation 108:1238-1245.

Address correspondence to: Christopher S. Rabender, Virginia Commonwealth University, $401 \mathrm{~N}$ College St, Rm 334, PO Box 980058, Richmond, VA 23298. E-mail: csrabender@vcu.edu 